International Journal of Pure and Applied Mathematics

Volume 110 No. 3 2016, 447-462

ISSN: 1311-8080 (printed version); ISSN: 1314-3395 (on-line version)

url: http://www.ijpam.eu

doi: 10.12732 /ijpam.v110i3.6

ijpam.eu

\title{
A MODEL FOR THE ADVERSE EFFECT OF TWO TOXICANTS CAUSING DEFORMITY IN A SUBCLASS OF A BIOLOGICAL SPECIES
}

\author{
Anuj Kumar Agarwal ${ }^{1 \S}$, A.W. Khan ${ }^{2}$, A.K. Agrawal $^{3}$ \\ ${ }^{1,2}$ Department of Mathematics \\ Integral University \\ Lucknow, INDIA \\ ${ }^{3}$ Department of Mathematics \\ Amity University \\ Lucknow, INDIA
}

\begin{abstract}
We have designed and analyzed a 6-dimensional mathematical model to study the effect of two toxicants on a biological species. A subclass of this biological species is badly affected by these toxins. This subclass of species shows deformity and is assumed to be not capable in further reproduction. We have assumed that one toxicant is being discharged into the environment by the biological species and the other toxicant is being emitted into the environment by some external sources. Using stability analysis and hopf-bifurcation theory, we have seen, when toxicant level (emitted by species) or/and toxicant level (emitted by external sources) increase, total density of species decreases, the density of the subclass of species increases. After crossing a critical concentration level of each toxicant, total density and density of the subclass of species start oscillations and show hopf-bifurcation.
\end{abstract}

AMS Subject Classification: 34C60, 37L10, 92D25, 93A30

Key Words: mathematical model, two toxicants, deformity, Hopf-bifurcation

Received: July 26, 2016

Revised: $\quad$ September 5, 2016

Published: November 5, 2016

$\S_{\text {Correspondence author }}$ (c) 2016 Academic Publications, Ltd. url: www.acadpubl.eu 


\section{Introduction}

Many researchers have proposed and analyzed mathematical models to study the effect of toxicants on biological species ([2], [8]). The research works have been carried out for different cases such as effect of a single toxicant, more than one toxicant, on a biological species or two biological species with a competition of thrives in the same environment etc. Further, a highly important phenomenon that is after-effect of toxicants a subclass of biological species shows deformity, was studied ([1], [5]). Agrawal et al. [1] proposed a model to study the effect of a single toxicant emitted from some external sources, on a biological species in which a subclass of biological species having abnormal symptoms and not capable in reproduction. In addition, Kumar et al. [5] proposed and analyzed a modified model to study the effect of a toxicant on a produced by the biological species itself, in which a subclass of biological species is severely affected.

Now, we propose and analyze a mathematical model to study the effect of two toxicants on a biological species. Here, we have assumed that one toxicant is being discharged into the environment by the biological species (which is very relevant when we consider human population) and the other toxicant is being emitted by some external source. We have assumed that the biological species gets adversely affected by these toxicants and even some members of the species show abnormal symptoms such as deformity, reduced reproduction capability etc.

\section{Mathematical Model}

We assume a biological species with population density $N(t)$ is logistically growing and surviving in that kind of environment which consist two different toxicants having concentrations $T_{1}(t) \& T_{2}(t)$ at time $t$. The first one toxicant $T_{1}(t)$ is being produced by the species itself and the second one toxicant $T_{2}(t)$ is released in the environment by other external sources. Let $U_{1}(t) \& U_{2}(t)$ are the concentrations at which toxicants of environmental concentrations $T_{1}(t) \&$ $T_{2}(t)$ are uptaken by the biological species respectively. A subclass of species with population density $N_{D}(t)$ is showing deformity as incapability of reproduction. Also, we assume $N_{A}(t)$ is remained density of $N(t)$ which is affected by toxicants but free from deformity. By assuming these facets, we proposed the following mathematical model: 


$$
\begin{aligned}
\frac{d N_{A}}{d t} & =(b-d) N_{A}-\left(r_{1} U_{1}+r_{2} U_{2}\right) N_{A}-\frac{r N_{A} N}{K\left(T_{1}, T_{2}\right)} \\
\frac{d N_{D}}{d t} & =\left(r_{1} U_{1}+r_{2} U_{2}\right) N_{A}-\frac{r N_{D} N}{K\left(T_{1}, T_{2}\right)}-(\alpha+d) N_{D} \\
\frac{d T_{1}}{d t} & =\lambda N-\delta_{1} T_{1}-\gamma_{1} T_{1} N+\pi_{1} \nu_{1} N U_{1}, \\
\frac{d T_{2}}{d t} & =Q-\delta_{2} T_{2}-\gamma_{2} T_{2} N+\pi_{2} \nu_{2} N U_{2}, \\
\frac{d U_{1}}{d t} & =\gamma_{1} T_{1} N-\beta_{1} U_{1}-\nu_{1} N U_{1}, \quad(1) \\
\frac{d U_{2}}{d t} & =\gamma_{2} T_{2} N-\beta_{2} U_{2}-\nu_{2} N U_{2}, \quad T_{i}(0) \geq 0, \quad U_{i}(0) \geq c_{i} N(0), \\
N(t)=N_{A}(t)+N_{D}(t), \quad N_{A}(0), N_{D}(0) \geq 0, & \quad \forall i=1,2 .
\end{aligned}
$$

Here, $r$ is the natural growth rate of species (i.e. the difference of natural birth rate $b$ and natural death rate $d)$. The toxicants of concentrations $T_{1}(t)$ $\& T_{2}(t)$ are discharged by the species $N(t)$ at the rate $\lambda$ and emitted by other external sources at constant rate $Q$. (For $i=1,2) T_{i}(t)$ is being depleted at the rate $\delta_{i}$ due to some natural factors. $\gamma_{i}$ is the rate at which $T_{i}(t)$ is being uptaken by the species. $\beta_{i}$ is the natural depletion rate of uptake concentration of toxicant $U_{i}(t)$. Due to the effect of uptake concentrations of toxicants $U_{i}(t)$, species is being incapable in reproduction at the rate $r_{i}$. Because of high toxicities in the environment, deformed species is dying out at the rate $\alpha . \nu_{i}$ is the depletion rate of $U_{i}(t)$ due to decay of some members of species $N(t)$ and a fraction $\pi_{i}$ of this decay re-entering into the environments and increasing $T_{i}(t)$. The constants $c_{i}>0$ are the proportionality constants to determine initial concentration of uptake toxicants. $K\left(T_{1}, T_{2}\right)$ is the maximum density which the environment can retain and it decreases when $T_{1}$ or/and $T_{2}$ increase.

Therefore

$$
\begin{aligned}
& K(0,0)=K_{0}>0, \quad K\left(T_{1}, T_{2}\right)>0, \\
& \frac{\partial K\left(T_{1}, T_{2}\right)}{\partial T_{1}}<0, \quad \frac{\partial K\left(T_{1}, T_{2}\right)}{\partial T_{2}}<0, \quad \text { for } \quad T_{1}, T_{2}>0
\end{aligned}
$$

where $K_{0}$ is the maximum density of species in a toxicants free environment. 
Using the fact that $N(t)=N_{A}(t)+N_{D}(t)$, the model (1) convert into following model which is free from $N_{A}(t)$,

$$
\begin{aligned}
\frac{d N}{d t} & =r N-\frac{r N^{2}}{K\left(T_{1}, T_{2}\right)}-(\alpha+b) N_{D} \\
\frac{d N_{D}}{d t} & =\left(r_{1} U_{1}+r_{2} U_{2}\right)\left(N-N_{D}\right)-\frac{r N_{D} N}{K\left(T_{1}, T_{2}\right)}-(\alpha+d) N_{D} \\
\frac{d T_{1}}{d t} & =\lambda N-\delta_{1} T_{1}-\gamma_{1} T_{1} N+\pi_{1} \nu_{1} N U_{1} \\
\frac{d T_{2}}{d t} & =Q-\delta_{2} T_{2}-\gamma_{2} T_{2} N+\pi_{2} \nu_{2} N U_{2} \\
\frac{d U_{1}}{d t} & =\gamma_{1} T_{1} N-\beta_{1} U_{1}-\nu_{1} N U_{1} \\
\frac{d U_{2}}{d t} & =\gamma_{2} T_{2} N-\beta_{2} U_{2}-\nu_{2} N U_{2} .
\end{aligned}
$$

The region $\Omega$ attracts all the positive solution of reduced model (3), as follows

$$
\begin{aligned}
& \Omega=\left\{\left(N, N_{D}, T_{1}, T_{2}, U_{1}, U_{2}\right): 0 \leq N \leq K_{0}, 0 \leq N_{D} \leq\right. \\
& \left.\frac{\left(r_{1}+r_{2}\right)\left(\lambda K_{0}+Q\right) K_{0}}{\left(r_{1}+r_{2}\right)\left(\lambda K_{0}+Q\right)+\delta_{m}(\alpha+d)}, 0 \leq T_{1}+T_{2}+U_{1}+U_{2} \leq \frac{\left(\lambda K_{0}+Q\right)}{\delta_{m}}\right\}
\end{aligned}
$$

where $\delta_{m}=\min \left(\delta_{1}, \delta_{2}, \beta_{1}, \beta_{2}\right)$.

\section{Equilibrium Points}

The model (3) possesses two non-negative equilibrium points $E_{1}\left(0,0,0, \frac{Q}{\delta_{2}}, 0,0\right)$ and $E_{2}\left(N^{*}, N_{D}^{*}, T_{1}^{*}, T_{2}^{*}, U_{1}^{*}, U_{2}^{*}\right)$. It is obvious that $E_{1}$ exists, hence existence of $E_{1}$ is omitted.

Existence of $\boldsymbol{E}_{\mathbf{2}}$ : The equilibrium point $E_{2}$ containing the values $N^{*}, N_{D}^{*}$, $T_{1}^{*}, T_{2}^{*}, U_{1}^{*}$ and $U_{2}^{*}$ are the positive solutions of following non-linear equations,

$$
\begin{aligned}
N & =\frac{\left(r-r_{1} U_{1}-r_{2} U_{2}\right) K\left(T_{1}, T_{2}\right)}{r} \\
N_{D} & =\frac{\left(r_{1} U_{1}+r_{2} U_{2}\right) N K\left(T_{1}, T_{2}\right)}{r N+\left(r_{1} U_{1}+r_{2} U_{2}+\alpha+d\right) K\left(T_{1}, T_{2}\right)} \\
T_{1} & =\frac{\lambda N\left(\beta_{1}+\nu_{1} N\right)}{f_{1}(N)}=g_{1}(N)(\text { say })
\end{aligned}
$$




$$
\begin{aligned}
T_{2} & =\frac{Q\left(\beta_{2}+\nu_{2} N\right)}{f_{2}(N)}=g_{2}(N)(\text { say }) \\
U_{1} & =\frac{\lambda \gamma_{1} N^{2}}{f_{1}(N)}=h_{1}(N)(\text { say }) \\
U_{2} & =\frac{Q \gamma_{2} N}{f_{2}(N)}=h_{2}(N)(\text { say }) \\
\text { where } \quad f_{1}(N) & =\delta_{1} \beta_{1}+\left(\gamma_{1} \beta_{1}+\delta_{1} \nu_{1}\right) N+\gamma_{1} \nu_{1}\left(1-\pi_{1}\right) N^{2} \\
f_{2}(N) & =\delta_{2} \beta_{2}+\left(\gamma_{2} \beta_{2}+\delta_{2} \nu_{2}\right) N+\gamma_{2} \nu_{2}\left(1-\pi_{2}\right) N^{2}
\end{aligned}
$$

The Eqs. (4c \& 4d) explain that the concentrations of both toxicants $T_{1} \&$ $T_{2}$ are directly dependent on the discharge rates $\lambda$ (by biological species) \& $Q$ (by external sources). Hence $K\left(T_{1}, T_{2}\right)$ decreases as $T_{1}($ or $\lambda)$ or $/$ and $T_{2}$ (or $Q$ ) increase.

Let

$$
F(N)=r N-\left(r-r_{1} h_{1}(N)-r_{2} h_{2}(N)\right) K\left(g_{1}(N), g_{2}(N)\right)
$$

at $N=0$,

$$
F(0)=-r K\left(0, \frac{Q}{\delta_{2}}\right)<0
$$

at $N=K_{0}$

$$
F\left(K_{0}\right)=r K_{0}-\left(r-r_{1} h_{1}\left(K_{0}\right)-r_{2} h_{2}\left(K_{0}\right)\right) K\left(g_{1}\left(K_{0}\right), g_{2}\left(K_{0}\right)\right)>0
$$

Eqs. (6 \& 7) guaranteed that at least one root exist between $0 \& K_{0}$ of $F(N)=$ 0 .

Uniqueness of $\boldsymbol{E}_{\mathbf{2}}: F(N)=0$ has a unique root $N^{*}$, if

$$
\begin{aligned}
\frac{d F}{d N}=\left[r+K\left(g_{1}(N), g_{2}(N)\right)\left\{r_{1} \frac{d h_{1}}{d N}+r_{2} \frac{d h_{2}}{d N}\right\}\right. & \\
& \left.-\left(r-r_{1} h_{1}(N)-r_{2} h_{2}(N)\right)\left\{\frac{\partial K}{\partial T_{1}} \frac{d g_{1}}{d N}+\frac{\partial K}{\partial T_{2}} \frac{d g_{2}}{d N}\right\}\right]>0
\end{aligned}
$$

As we know, from Eq. (2)

$$
\frac{\partial K\left(T_{1}, T_{2}\right)}{\partial T_{1}}<0, \quad \frac{\partial K\left(T_{1}, T_{2}\right)}{\partial T_{2}}<0
$$

And from the "Eqs. (4c-4f)"

$$
\frac{d g_{1}}{d N}=\frac{\lambda}{f_{1}^{2}(N)}\left[\delta_{1}\left(\beta_{1}+\nu_{1} N\right)^{2}+\gamma_{1} \nu_{1} \pi_{1} \beta_{1} N^{2}\right]>0
$$




$$
\begin{aligned}
\frac{d g_{2}}{d N} & =-\frac{Q \gamma_{2}}{f_{2}^{2}(N)}\left[\left(1-\pi_{2}\right)\left(\beta_{2}+\nu_{2} N\right)^{2}+\pi_{2} \beta_{2}^{2}\right]<0 \\
\frac{d h_{1}}{d N} & =\frac{\lambda \gamma_{1} N}{f_{1}^{2}(N)}\left[2 \delta_{1} \beta_{1}+\left(\gamma_{1} \beta_{1}+\delta_{1} \nu_{1}\right) N\right]>0 \\
\frac{d h_{2}}{d N} & =\frac{Q \gamma_{2}}{f_{2}^{2}(N)}\left[\delta_{2} \beta_{2}-\gamma_{2} \nu_{2}\left(1-\pi_{2}\right) N^{2}\right] \\
& \left.>0 \text { only when } \delta_{2} \beta_{2}>\gamma_{2} \nu_{2}\left(1-\pi_{2}\right) N^{2}\right\}
\end{aligned}
$$

Eqs. (9a-9d) implies that $\frac{d F}{d N}>0$ as well as root $N^{*}$ will be unique, if

$$
\begin{aligned}
\delta_{2} \beta_{2} & >\gamma_{2} \nu_{2}\left(1-\pi_{2}\right) N^{2} \\
\frac{\partial K\left(T_{1}, T_{2}\right)}{\partial T_{1}} \frac{d g_{1}}{d N} & >\frac{\partial K\left(T_{1}, T_{2}\right)}{\partial T_{2}} \frac{d g_{2}}{d N}
\end{aligned}
$$

Hence, we can see that if conditions (10a-10b) hold then $F(N)=0$ must have a unique root $N^{*}$ in the interval $\left[0, K_{0}\right]$. As a result of root $N^{*}$, we can simply find out other points $N_{D}^{*}, T_{1}^{*}, T_{2}^{*}, U_{1}^{*} \& U_{2}^{*}$ of $E_{2}$ using Eqs. (4a-4h).

\section{Dynamical Behavior}

\subsection{Dynamical Behavior Corresponding to $E_{1}$}

The Jacobian matrix $M_{1}$ (say) of the model (3) at the equilibrium point $E_{1}$, we have

$$
M_{1}=\left[\begin{array}{cccccc}
r & -(\alpha+b) & 0 & 0 & 0 & 0 \\
0 & -(\alpha+d) & 0 & 0 & 0 & 0 \\
\lambda & 0 & -\delta_{1} & 0 & 0 & 0 \\
-\frac{\gamma_{2} Q}{\delta_{2}} & 0 & 0 & -\delta_{2} & 0 & 0 \\
0 & 0 & 0 & 0 & -\beta_{1} & 0 \\
\frac{\gamma_{2} Q}{\delta_{2}} & 0 & 0 & 0 & 0 & -\beta_{2}
\end{array}\right]
$$

Here, the eigenvalues of Jacobian matrix $M_{1}$ are $r,-(\alpha+d),-\delta_{1},-\delta_{2},-\beta_{1}$ and $-\beta_{2}$. Consequently, $E_{1}$ is a saddle point locally unstable manifold in $N-$ direction and locally stable manifold in $N_{D}-T_{1}-T_{2}-U_{1}-U_{2}$ space. 


\subsection{Dynamical Behavior Corresponding to $E_{2}$}

The Jacobian matrix $M_{2}$ (say) of the model (3) at the equilibrium point $E_{2}$, we have

$$
M_{2}=\left[\begin{array}{cccccc}
m_{11} & m_{12} & m_{13} & m_{14} & 0 & 0 \\
m_{21} & m_{22} & m_{23} & m_{24} & m_{25} & m_{26} \\
m_{31} & 0 & m_{33} & 0 & m_{35} & 0 \\
m_{41} & 0 & 0 & m_{44} & 0 & m_{46} \\
m_{51} & 0 & m_{53} & 0 & m_{55} & 0 \\
m_{61} & 0 & 0 & m_{64} & 0 & m_{66}
\end{array}\right] \text {, }
$$

where

$$
\begin{gathered}
m_{11}=-r\left\{\frac{2 N^{*}}{K\left(T_{1}^{*}, T_{2}^{*}\right)}-1\right\}, m_{12}=-(\alpha+b), m_{13}=\frac{r N^{* 2}}{K^{2}\left(T_{1}^{*}, T_{2}^{*}\right)}\left[\frac{\partial K}{\partial T_{1}}\right]_{E_{2}}, \\
m_{14}=\frac{r N^{* 2}}{K^{2}\left(T_{1}^{*}, T_{2}^{*}\right)}\left[\frac{\partial K}{\partial T_{2}}\right]_{E_{2}}, m_{21}=r_{1} U_{1}^{*}+r_{2} U_{2}^{*}-\frac{r N_{D}^{*}}{K\left(T_{1}^{*}, T_{2}^{*}\right)}, \\
m_{22}=-\left(r_{1} U_{1}^{*}+r_{2} U_{2}^{*}\right) \frac{N^{*}}{N_{D}^{*}}, m_{23}=\frac{r N^{*} N_{D}^{*}}{K^{2}\left(T_{1}^{*}, T_{2}^{*}\right)}\left[\frac{\partial K}{\partial T_{1}}\right]_{E_{2}}, \\
m_{24}=\frac{r N^{*} N_{D}^{*}}{K^{2}\left(T_{1}^{*}, T_{2}^{*}\right)}\left[\frac{\partial K}{\partial T_{2}}\right]_{E_{2}}, m_{25}=r_{1}\left(N^{*}-N_{D}^{*}\right), m_{26}=r_{2}\left(N^{*}-N_{D}^{*}\right), \\
m_{31}=\lambda-\gamma_{1} T_{1}^{*}+\pi_{1} \nu_{1} U_{1}^{*}, m_{33}=-\left(\delta_{1}+\gamma_{1} N^{*}\right), m_{35}=\pi_{1} \nu_{1} N^{*}, \\
m_{41}=-\gamma_{2} T_{2}^{*}+\pi_{2} \nu_{2} U_{2}^{*}, m_{44}=-\frac{\left(Q+\pi_{2} \nu_{2} N^{*} U_{2}^{*}\right)}{T_{2}^{*}}, m_{46}=\pi_{2} \nu_{2} N^{*}, \\
m_{51}=\gamma_{1} T_{1}^{*}-\nu_{1} U_{1}^{*}, m_{53}=\gamma_{1} N^{*}, m_{55}=-\left(\beta_{1}+\nu_{1} N^{*}\right), m_{61}=\gamma_{2} T_{2}^{*}-\nu_{2} U_{2}^{*}, \\
m_{64}=\gamma_{2} N^{*}, m_{66}=-\left(\beta_{2}+\nu_{2} N^{*}\right) .
\end{gathered}
$$

The characteristic equation of matrix $M_{2}$ can be written as:

$$
p(x)=x^{6}+c_{1} x^{5}+c_{2} x^{4}+c_{3} x^{3}+c_{4} x^{2}+c_{5} x+c_{6}
$$

The coefficients $c_{1}, c_{2}, c_{3}, c_{4}, c_{5}$ and $c_{6}$ are defined in Appendix A.

Using the Routh-Hurwitz Criterion, all the roots of the polynomial $p(x)$ are either negative or with negative real parts iff

$$
\begin{gathered}
c_{k}>0, \quad(k=1,6) \\
H_{2}=c_{1} c_{2}-c_{3}>0
\end{gathered}
$$




$$
\begin{gathered}
H_{3}=c_{1} c_{2} c_{3}+c_{1} c_{5}-c_{3}^{2}-c_{1}^{2} c_{4}>0 \\
H_{4}=\left(c_{1} c_{2}-c_{3}\right)\left(c_{3} c_{4}-c_{2} c_{5}\right)+\left(c_{1} c_{2}-c_{3}\right) c_{1} c_{6}-\left(c_{1} c_{4}-c_{5}\right)^{2}>0 \\
H_{5}=c_{3}\left(c_{1} c_{2}-c_{3}\right)\left(c_{4} c_{5}-c_{3} c_{6}\right)+c_{3} c_{5}\left(c_{2} c_{5}-c_{1} c_{6}\right)+c_{1} c_{3} c_{6}\left(c_{1} c_{4}\right. \\
\left.-2 c_{5}\right)-c_{1}\left(c_{2} c_{5}-c_{1} c_{6}\right)^{2}-c_{5}\left(c_{1} c_{4}-c_{5}\right)^{2}>0
\end{gathered}
$$

Now, we can state the following result on the local asymptotically stablility of the equilibrium point $E_{2}$.

Theorem 1. The equilibrium point $E_{2}$ is locally asymptotically stable under the conditions (12a-12e).

We check the existence of Hopf-bifurcation ([3], [6]) for the equilibrium point $E_{2}$ by taking $\lambda$ and $Q$ as bifurcation parameters. The necessary and sufficient conditions for the existence of Hopf-bifurcation in model (3): Eigenvalues $x_{k}=R_{k}+i I_{k},(k=1, \cdots, 6)$ of the Jacobian matrix $M_{2}$ have a pair of purely imaginary eigenvalues and others eigenvalues have negative real parts (i.e., $R_{1}, R_{2}=0, I_{1}=-I_{2} \neq 0 \& R_{3}, R_{4}, R_{5}, R_{6}<0$ ) at the critical values of parameters $\lambda=\lambda^{*}$ and $Q=Q^{*}$.

Now, according to the Liu's criterion [7] model (3) undergoes a Hopfbifurcation at the critical value of $\lambda=\lambda^{*}>0$, if following conditions hold:

$$
\begin{aligned}
& {\left[c_{k}\right]_{\lambda=\lambda^{*}}>0 \text { for }(k=1, \cdots, 6) } \\
{\left[H_{2}\right]_{\lambda=\lambda^{*}}=} & {\left[c_{1} c_{2}-c_{3}\right]_{\lambda=\lambda^{*}}>0 } \\
{\left[H_{3}\right]_{\lambda=\lambda^{*}}=} & {\left[c_{1} c_{2} c_{3}+c_{1} c_{5}-c_{3}^{2}-c_{1}^{2} c_{4}\right]_{\lambda=\lambda^{*}}>0 } \\
{\left[H_{4}\right]_{\lambda=\lambda^{*}}=} & {\left[\left(c_{1} c_{2}-c_{3}\right)\left(c_{3} c_{4}-c_{2} c_{5}\right)+\left(c_{1} c_{2}-c_{3}\right) c_{1} c_{6}\right.} \\
& \left.-\left(c_{1} c_{4}-c_{5}\right)^{2}\right]_{\lambda=\lambda^{*}}>0 \\
{\left[H_{5}\right]_{\lambda=\lambda^{*}}=} & {\left[c_{3}\left(c_{1} c_{2}-c_{3}\right)\left(c_{4} c_{5}-c_{3} c_{6}\right)+c_{3} c_{5}\left(c_{2} c_{5}-c_{1} c_{6}\right)\right.} \\
& +c_{1} c_{3} c_{6}\left(c_{1} c_{4}-2 c_{5}\right)-c_{1}\left(c_{2} c_{5}-c_{1} c_{6}\right)^{2} \\
& \left.-c_{5}\left(c_{1} c_{4}-c_{5}\right)^{2}\right]_{\lambda=\lambda^{*}}=0 \\
& \neq 0, \quad \text { for } k=1,2 \\
{\left[\frac{d R_{k}}{d \lambda}\right]_{\lambda=\lambda^{*}} } &
\end{aligned}
$$

Using A New Detecting Method For Conditions of Existence of Hopf-bifurcation [4], we get the last condition "(13f)" as follows:

$$
\left[\frac{d R}{d \lambda}\right]_{\lambda=\lambda^{*}}=\left[\frac{1}{B} \frac{d H_{5}}{d \lambda}\right]_{\lambda=\lambda^{*}} \neq 0
$$


where, $H_{5}$ defined as "Eq. (13e)" and

$$
B=2\left\{c_{1}\left(c_{1} c_{4}-c_{5}\right)-c_{3}\left(c_{1} c_{2}-c_{3}\right)\right\}\left|\begin{array}{ccccc}
-c_{1} & 1 & 1 & 0 & 0 \\
c_{2}-2 A & -c_{1} & 0 & 1 & 0 \\
c_{1} A-c_{3} & c_{2}-A & A & 0 & 1 \\
A^{2}-c_{2} A+c_{4} & c_{1} A-c_{3} & 0 & A & 0 \\
0 & A^{2}-c_{2} A+c_{4} & 0 & 0 & A
\end{array}\right|
$$

also $A=\frac{c_{3} c_{5}+c_{1}^{2} c_{6}-c_{1} c_{2} c_{5}}{c_{1}^{2} c_{4}-c_{1} c_{5}-c_{1} c_{2} c_{3}+c_{3}^{2}}$.

Hence, we can state the following result.

Theorem 2. The model (3) undergoes a Hopf-bifurcation corresponding to the equilibrium point $E_{2}$, if $\lambda$ crosses the critical value $\lambda^{*}>0$ such that

1. $\left[c_{k}\right]_{\lambda=\lambda^{*}}>0$ for $(k=1, \cdots, 6)$

2. $\left[H_{2}\right]_{\lambda=\lambda^{*}}=\left[c_{1} c_{2}-c_{3}\right]_{\lambda=\lambda^{*}}>0$

3. $\left[H_{3}\right]_{\lambda=\lambda^{*}}=\left[c_{1} c_{2} c_{3}+c_{1} c_{5}-c_{3}^{2}-c_{1}^{2} c_{4}\right]_{\lambda=\lambda^{*}}>0$

4. $\left[H_{4}\right]_{\lambda=\lambda^{*}}=\left[\left(c_{1} c_{2}-c_{3}\right)\left(c_{3} c_{4}-c_{2} c_{5}\right)+\left(c_{1} c_{2}-c_{3}\right) c_{1} c_{6}\right.$ $\left.-\left(c_{1} c_{4}-c_{5}\right)^{2}\right]_{\lambda=\lambda^{*}}>0$

5. $\left[H_{5}\right]_{\lambda=\lambda^{*}}=\left[c_{3}\left(c_{1} c_{2}-c_{3}\right)\left(c_{4} c_{5}-c_{3} c_{6}\right)+c_{3} c_{5}\left(c_{2} c_{5}-c_{1} c_{6}\right)\right.$ $+c_{1} c_{3} c_{6}\left(c_{1} c_{4}-2 c_{5}\right)-c_{1}\left(c_{2} c_{5}-c_{1} c_{6}\right)^{2}$ $\left.-c_{5}\left(c_{1} c_{4}-c_{5}\right)^{2}\right]_{\lambda=\lambda^{*}}=0$

6. $\left[\frac{d R}{d \lambda}\right]_{\lambda=\lambda^{*}}=\left[\frac{1}{B} \frac{d H_{5}}{d \lambda}\right]_{\lambda=\lambda^{*}} \neq 0$

Similarly, the model (3) undergoes a Hopf-bifurcation at the critical value of $Q=Q^{*}>0$, if following theorem holds:

Theorem 3. The model (3) undergoes a Hopf-bifurcation corresponding to the equilibrium point $E_{2}$, if $Q$ crosses the critical value $Q^{*}>0$ such that

1. $\left[c_{k}\right]_{Q=Q^{*}}>0$ for $(k=1, \cdots, 6)$

2. $\left[H_{2}\right]_{Q=Q^{*}}=\left[c_{1} c_{2}-c_{3}\right]_{Q=Q^{*}}>0$

3. $\left[H_{3}\right]_{Q=Q^{*}}=\left[c_{1} c_{2} c_{3}+c_{1} c_{5}-c_{3}^{2}-c_{1}^{2} c_{4}\right]_{Q=Q^{*}}>0$ 
4. $\left[H_{4}\right]_{Q=Q^{*}}=\left[\left(c_{1} c_{2}-c_{3}\right)\left(c_{3} c_{4}-c_{2} c_{5}\right)+\left(c_{1} c_{2}-c_{3}\right) c_{1} c_{6}\right.$ $\left.-\left(c_{1} c_{4}-c_{5}\right)^{2}\right]_{Q=Q^{*}}>0$

5. $\left[H_{5}\right]_{Q=Q^{*}}=\left[c_{3}\left(c_{1} c_{2}-c_{3}\right)\left(c_{4} c_{5}-c_{3} c_{6}\right)+c_{3} c_{5}\left(c_{2} c_{5}-c_{1} c_{6}\right)\right.$ $+c_{1} c_{3} c_{6}\left(c_{1} c_{4}-2 c_{5}\right)-c_{1}\left(c_{2} c_{5}-c_{1} c_{6}\right)^{2}$ $\left.-c_{5}\left(c_{1} c_{4}-c_{5}\right)^{2}\right]_{Q=Q^{*}}=0$

6. $\left[\frac{d R}{d Q}\right]_{Q=Q^{*}}=\left[\frac{1}{B} \frac{d H_{5}}{d Q}\right]_{Q=Q^{*}} \neq 0$

where $B$ defined as equation (15).

The above "Theorems: $2 \& 3$ " characterize that equilibrium point $E_{2}$ become unstable and show Hopf-bifurcation when the emission rate of toxicant by the biological species $\lambda$ and the discharge rate of toxicant from some external sources $Q$ crosses the critical values $\lambda^{*}$ and $Q^{*}$ correspondingly.

\section{Numerical Simulation}

In this section, we provide numerical simulation to clarify the dynamic behavior of solutions of model (3). Let us assume the carrying capacity function as

$$
K\left(T_{1}, T_{2}\right)=K_{0}-\frac{b_{11} T_{1}}{1+b_{12} T_{1}}-\frac{b_{21} T_{2}}{1+b_{22} T_{2}}
$$

and a set of parameters as:

$$
\begin{aligned}
& b=0.044, d=0.001, r_{1}=0.035, r_{2}=0.032, \lambda=0.001, \alpha=0.0008, \\
& Q=0.005, \delta_{1}=0.04, \delta_{2}=0.09, \gamma_{1}=0.00045, \gamma_{2}=0.001, \pi_{1}=0.05, \\
& \pi_{2}=0.06, \nu_{1}=0.00004, \nu_{2}=0.00003, \beta_{1}=0.01, \beta_{2}=0.006, \\
& K_{0}=10.0, b_{11}=0.2, b_{12}=1.0, b_{21}=0.1, b_{22}=2.0
\end{aligned}
$$

It is noted that for these values of the parameters, the local stability conditions (12a-12e). corresponding to the equilibrium $E_{2}$ are satisfied. Now, we see the variations of two important parameters $\lambda$ and $Q$ on total population density $N$ and the deformed population density $N_{D}$.

\subsection{Parameter $\lambda$}

We have considered different values of the emission rate of toxicant $\lambda$ by the biological species and assumed remaining parameters same as in "Eq. (16b)". 
The "Fig. 1" show that for small values of $\lambda$, the density of total population $N$ decreases and deformed population $N_{D}$ firstly increase then decrease as $N$ decreases, and both densities became stable at their equilibrium levels. But, for large value $\lambda$ (for $\lambda=0.200$ ) both densities start oscillating and do not settle down to their equilibrium levels. So, there may exist a Hopf-bifurcation corresponding to the parameter $\lambda$.
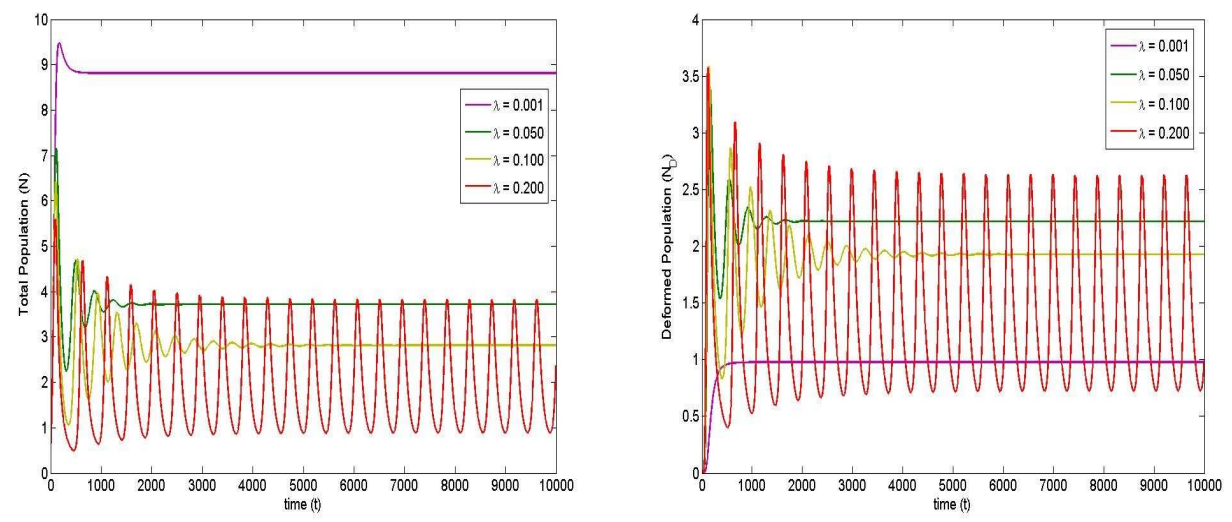

Figure 1: Time series graph for total and deformed population respect to the emission rate $\lambda$.
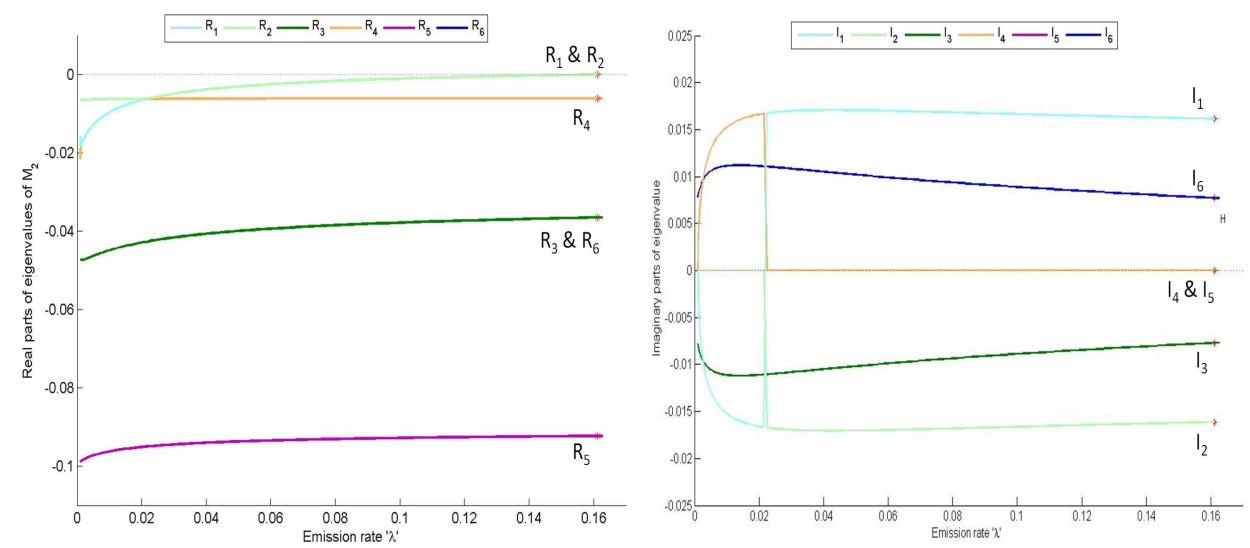

Figure 2: Real and Imaginary parts of eigenvalues of Jacobian matrix $M_{2}$ respect to the emission rate $\lambda$.

The "Fig. 2" show the variations in real and imaginary parts of eigenvalues 
of Jacobian matrix $M_{2}$ on increasing $\lambda$. In this figure, we can see that the real parts of all eigenvalues (i.e. $\left.R_{i}<0, i=1, \cdots, 6\right)$ of Jacobian matrix $M_{2}$ are negative for $\lambda<\lambda^{*}(=0.16114)$. At $\lambda=\lambda^{*}$ two eigenvalues of $M_{2}$ become purely imaginary (i.e. $R_{1}=R_{2}=0 \& I_{1}=-I_{2} \neq 0$ ) and other four eigenvalues have negative real parts (i.e. $R_{i}<0, i=3, \cdots, 6$ ). Thus, if the emission rate $\lambda$ is less than critical value $\lambda^{*}$, the model (3) is locally asymptotically stable. But at the critical value $\lambda^{*}$, the model (3) undergoes a Hopf-bifurcation.

\subsection{Parameter $Q$}

Here, we have considered different values of the emission rate of toxicant $Q$ by some external sources. Keeping all the other parameters same as in "Eq. 16b". The "Fig. 3" show that for small value of $Q$, density of total population $N$ decreases and deformed population $N_{D}$ firstly increase then decrease as $N$ decreases. Both densities initially oscillate and became stable at their equilibrium levels. But, for large value of $Q$ (for $Q=0.800$ ), both densities start oscillating and do not settle down to their equilibrium levels. Also, there may exist a Hopf-bifurcation corresponding to the parameter $Q$.
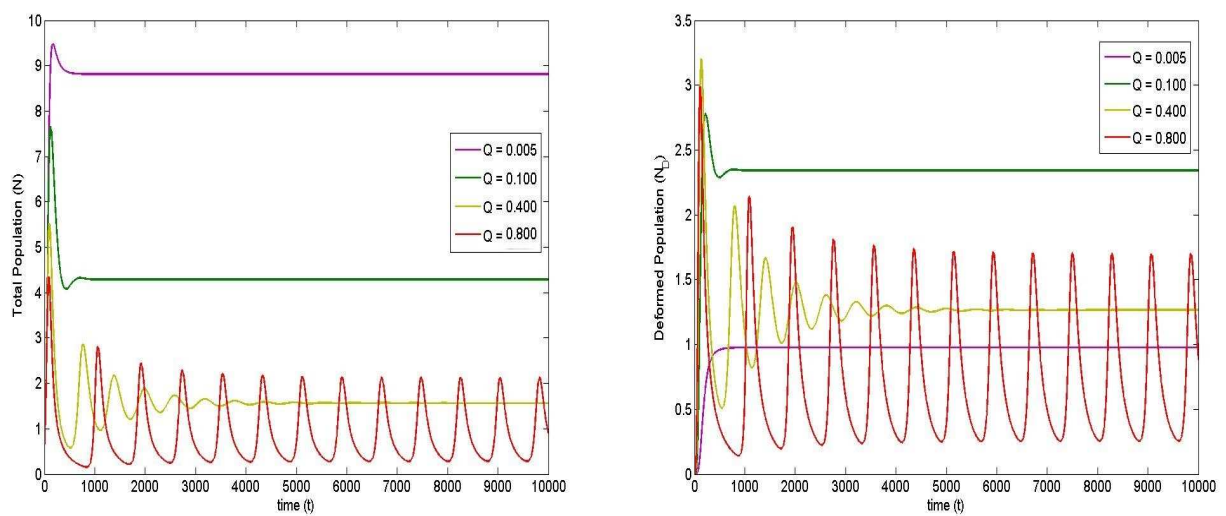

Figure 3: Time series graph for total and deformed population respect to the emission rate $Q$.

In the "Fig. 4", we have seen the changes in real and imaginary parts of eigenvalues of Jacobian matrix $M_{2}$ on increasing $Q$. The Fig.4 shows that all the eigenvalues of Jacobian matrix $M_{2}$ have negative real parts (i.e. $R_{i}<0$, $i=1, \cdots, 6)$ for each $Q<Q^{*}(=0.61797)$. At $Q=Q^{*}$, two eigenvalues of $M_{2}$ become purely imaginary (i.e. $R_{1}=R_{2}=0 \& I_{1}=-I_{2} \neq 0$ ) and remaining 
four eigenvalues have negative real parts (i.e. $R_{i}<0, i=3, \cdots, 6$ ). Thus, model system (3) is locally asymptotically stable for each $Q<Q^{*}$. At $Q=Q^{*}$, the model system (3) undergoes a Hopf-bifurcation.
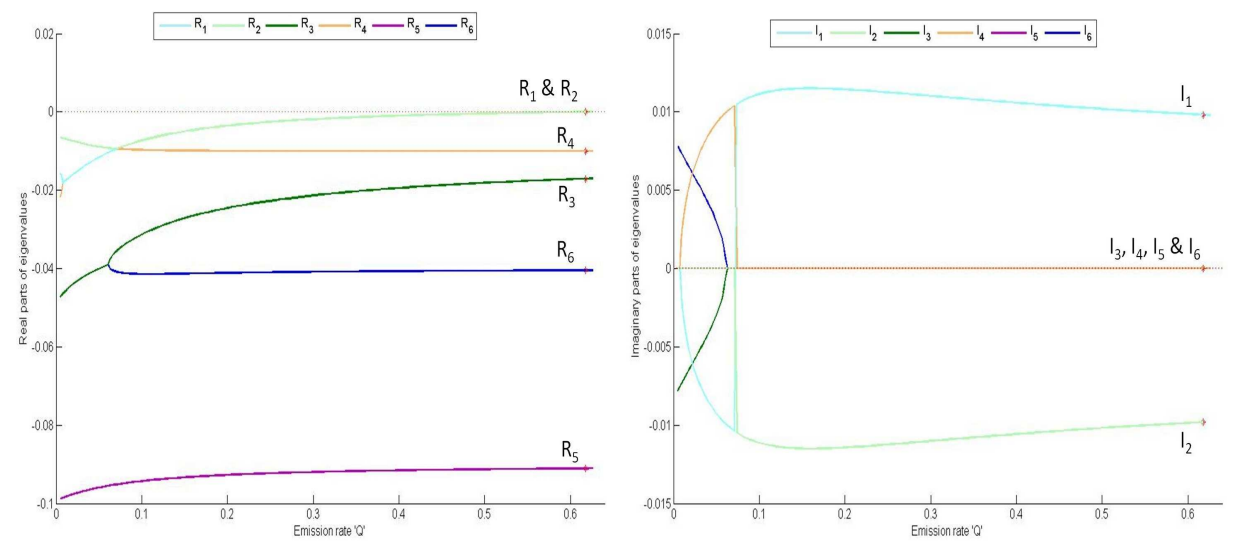

Figure 4: Real and Imaginary parts of eigenvalues of Jacobian matrix $M_{2}$ respect to the emission rate $Q$.

\section{Conclusion}

This paper is based on effect of two toxicants on a logistically growing biologically species. We have proposed and analyzed a mathematical model for the case in which a subclass of biological species which is badly affected by these toxicants, shows deformity such as reproduction failure. We have assumed that one toxicant is being discharged into the environment by the biological species and the other toxicant is being emitted into the environment by some external sources. The qualitative analysis of model shows that when toxicant level (emitted by species) or/and toxicant level (emitted by external sources) increase, total density of species decreases, the density of the subclass of species firstly increases and then it decreases as total density decreases. After crossing a critical concentration level of each toxicant, total density and density of the subclass of species starts stable periodic oscillation around their equilibria and show supercritical hopf-bifurcation. 


\section{Appendix A: Coefficients of Characteristic Equation (11)}

$$
\begin{aligned}
& c_{1}=-\left(m_{11}+m_{22}+m_{33}+m_{44}+m_{55}+m_{66}\right), \\
& c_{2}=\left(m_{11}+m_{22}\right)\left(m_{55}+m_{66}\right)+m_{22}\left(m_{33}+m_{44}\right)+\left(m_{33}+m_{55}\right)\left(m_{44}+m_{66}\right) \\
& +\left|\begin{array}{ll}
m_{11} & m_{12} \\
m_{21} & m_{22}
\end{array}\right|+\left|\begin{array}{ll}
m_{11} & m_{13} \\
m_{31} & m_{33}
\end{array}\right|+\left|\begin{array}{ll}
m_{11} & m_{14} \\
m_{41} & m_{44}
\end{array}\right|+\left|\begin{array}{ll}
m_{33} & m_{35} \\
m_{53} & m_{55}
\end{array}\right|+\left|\begin{array}{ll}
m_{44} & m_{46} \\
m_{64} & m_{66}
\end{array}\right|, \\
& c_{3}=m_{14} m_{41} m_{33}-m_{11} m_{55} m_{66}-m_{12}\left(m_{25} m_{51}+m_{26} m_{61}\right) \\
& -m_{22}\left(m_{33}+m_{55}\right)\left(m_{44}+m_{66}\right)+m_{13}\left|\begin{array}{ll}
m_{31} & m_{35} \\
m_{51} & m_{55}
\end{array}\right|+m_{14}\left|\begin{array}{ll}
m_{41} & m_{46} \\
m_{61} & m_{66}
\end{array}\right| \\
& -m_{31}\left|\begin{array}{ll}
m_{12} & m_{13} \\
m_{22} & m_{23}
\end{array}\right|-m_{41}\left|\begin{array}{ll}
m_{12} & m_{14} \\
m_{22} & m_{24}
\end{array}\right|-m_{55}\left|\begin{array}{ll}
m_{11} & m_{14} \\
m_{41} & m_{44}
\end{array}\right|-\left(m_{44}+m_{66}\right)\left|\begin{array}{ll}
m_{11} & m_{13} \\
m_{31} & m_{33}
\end{array}\right| \\
& -\left(m_{11}+m_{22}+m_{44}+m_{66}\right)\left|\begin{array}{ll}
m_{33} & m_{35} \\
m_{53} & m_{55}
\end{array}\right|-\left(m_{11}+m_{22}+m_{33}+m_{55}\right)\left|\begin{array}{ll}
m_{44} & m_{46} \\
m_{64} & m_{66}
\end{array}\right| \\
& -\left(m_{33}+m_{44}+m_{55}+m_{66}\right)\left|\begin{array}{cc}
m_{11} & m_{12} \\
m_{21} & m_{22}
\end{array}\right| \text {, } \\
& c_{4}=m_{12}\left\{m_{26} m_{61}\left(m_{33}+m_{55}\right)+m_{25} m_{51}\left(m_{44}+m_{66}\right)+m_{31}\left|\begin{array}{ll}
m_{23} & m_{25} \\
m_{53} & m_{55}
\end{array}\right|\right. \\
& \left.+m_{41}\left|\begin{array}{ll}
m_{24} & m_{26} \\
m_{64} & m_{66}
\end{array}\right|-m_{51}\left|\begin{array}{cc}
m_{23} & m_{25} \\
m_{33} & m_{35}
\end{array}\right|-m_{61}\left|\begin{array}{cc}
m_{24} & m_{26} \\
m_{44} & m_{46}
\end{array}\right|\right\} \\
& +m_{31}\left(m_{44}+m_{66}\right)\left|\begin{array}{ll}
m_{12} & m_{13} \\
m_{22} & m_{23}
\end{array}\right|+m_{41}\left(m_{33}+m_{55}\right)\left|\begin{array}{ll}
m_{12} & m_{14} \\
m_{22} & m_{24}
\end{array}\right| \\
& -m_{13}\left(m_{22}+m_{44}+m_{66}\right)\left|\begin{array}{ll}
m_{31} & m_{35} \\
m_{51} & m_{55}
\end{array}\right|-m_{14}\left(m_{22}+m_{33}+m_{55}\right)\left|\begin{array}{ll}
m_{41} & m_{46} \\
m_{61} & m_{66}
\end{array}\right| \\
& +\left\{m_{11} m_{55}+m_{22}\left(m_{33}+m_{55}\right)+\left|\begin{array}{ll}
m_{11} & m_{13} \\
m_{31} & m_{33}
\end{array}\right|\right\}\left|\begin{array}{ll}
m_{44} & m_{46} \\
m_{64} & m_{66}
\end{array}\right| \\
& +\left\{\left(m_{33}+m_{55}\right)\left(m_{44}+m_{66}\right)+\left|\begin{array}{ll}
m_{33} & m_{35} \\
m_{53} & m_{55}
\end{array}\right|+\left|\begin{array}{ll}
m_{44} & m_{46} \\
m_{64} & m_{66}
\end{array}\right|\right\}\left|\begin{array}{ll}
m_{11} & m_{12} \\
m_{21} & m_{22}
\end{array}\right| \\
& +\left\{m_{11} m_{66}+m_{22}\left(m_{44}+m_{66}\right)+\left|\begin{array}{cc}
m_{11} & m_{14} \\
m_{41} & m_{44}
\end{array}\right|+\left|\begin{array}{cc}
m_{44} & m_{46} \\
m_{64} & m_{66}
\end{array}\right|\right\}\left|\begin{array}{ll}
m_{33} & m_{35} \\
m_{53} & m_{55}
\end{array}\right| \text {, }
\end{aligned}
$$




$$
\begin{aligned}
& c_{5}=\left(m_{33}+m_{55}\right)\left\{m_{14} m_{22}\left|\begin{array}{ll}
m_{41} & m_{46} \\
m_{61} & m_{66}
\end{array}\right|+m_{61} m_{12}\left|\begin{array}{ll}
m_{24} & m_{26} \\
m_{44} & m_{46}
\end{array}\right|\right. \\
& \left.-m_{12} m_{41}\left|\begin{array}{ll}
m_{24} & m_{26} \\
m_{64} & m_{66}
\end{array}\right|\right\}+\left(m_{44}+m_{66}\right)\left\{m_{12} m_{51}\left|\begin{array}{ll}
m_{23} & m_{25} \\
m_{33} & m_{35}
\end{array}\right|\right. \\
& \left.-m_{12} m_{31}\left|\begin{array}{ll}
m_{23} & m_{25} \\
m_{53} & m_{55}
\end{array}\right|\right\}+m_{13}\left\{m_{22}\left(m_{44}+m_{66}\right)+\left|\begin{array}{ll}
m_{44} & m_{46} \\
m_{64} & m_{66}
\end{array}\right|\right\}\left|\begin{array}{ll}
m_{31} & m_{35} \\
m_{51} & m_{55}
\end{array}\right| \\
& -\left\{m_{12} m_{25} m_{51}+m_{31}\left|\begin{array}{ll}
m_{12} & m_{13} \\
m_{22} & m_{23}
\end{array}\right|\right\}\left|\begin{array}{ll}
m_{44} & m_{46} \\
m_{64} & m_{66}
\end{array}\right|-\left\{\left(m_{44}+m_{66}\right)\left|\begin{array}{ll}
m_{33} & m_{35} \\
m_{53} & m_{55}
\end{array}\right|\right. \\
& \left.+\left(m_{33}+m_{55}\right)\left|\begin{array}{ll}
m_{44} & m_{46} \\
m_{64} & m_{66}
\end{array}\right|\right\}\left|\begin{array}{ll}
m_{11} & m_{12} \\
m_{21} & m_{22}
\end{array}\right|-\left\{\left(m_{11}+m_{22}\right)\left|\begin{array}{ll}
m_{44} & m_{46} \\
m_{64} & m_{66}
\end{array}\right|\right. \\
& \left.+m_{12} m_{26} m_{61}+m_{41}\left|\begin{array}{ll}
m_{12} & m_{14} \\
m_{22} & m_{24}
\end{array}\right|-m_{14}\left|\begin{array}{ll}
m_{41} & m_{46} \\
m_{61} & m_{66}
\end{array}\right|\right\}\left|\begin{array}{ll}
m_{33} & m_{35} \\
m_{53} & m_{55}
\end{array}\right| \\
& c_{6}=\left|\begin{array}{ll}
m_{11} & m_{12} \\
m_{21} & m_{22}
\end{array}\right|\left|\begin{array}{ll}
m_{33} & m_{35} \\
m_{53} & m_{55}
\end{array}\right|\left|\begin{array}{cc}
m_{44} & m_{46} \\
m_{64} & m_{66}
\end{array}\right|+\left|\begin{array}{cc}
m_{12} & m_{14} \\
m_{22} & m_{24}
\end{array}\right|\left|\begin{array}{cc}
m_{33} & m_{35} \\
m_{53} & m_{55}
\end{array}\right|\left|\begin{array}{cc}
m_{41} & m_{46} \\
m_{61} & m_{66}
\end{array}\right| \\
& +\left|\begin{array}{ll}
m_{31} & m_{35} \\
m_{51} & m_{55}
\end{array}\right|\left|\begin{array}{ll}
m_{12} & m_{13} \\
m_{22} & m_{23}
\end{array}\right|\left|\begin{array}{cc}
m_{44} & m_{46} \\
m_{64} & m_{66}
\end{array}\right|-m_{12} m_{26}\left|\begin{array}{cc}
m_{41} & m_{44} \\
m_{61} & m_{64}
\end{array}\right|\left|\begin{array}{ll}
m_{33} & m_{35} \\
m_{53} & m_{55}
\end{array}\right| \\
& -m_{12} m_{25}\left|\begin{array}{ll}
m_{31} & m_{33} \\
m_{51} & m_{53}
\end{array}\right|\left|\begin{array}{cc}
m_{44} & m_{46} \\
m_{64} & m_{66}
\end{array}\right|
\end{aligned}
$$

where, $\left|\begin{array}{ll}m_{1} & m_{2} \\ m_{3} & m_{4}\end{array}\right|=m_{1} m_{4}-m_{2} m_{3}$.

\section{References}

[1] A.K. Agrawal, J.B. Shukla, Effect of a toxicant on a biological population causing severe symptoms on a subclass, South Pacific Journal of Pure and Applied Mathematics, 1, No. 1 (2012), 12-27.

[2] H.I. Freedman, J.B. Shukla, Models for the effect of toxicant in single species and predator prey systems, J. Math. Biol., 30 (1991), 15-30, doi: 10.1007/BF00168004.

[3] B.D. Hassard, N.D. Kazarinoff, Y.H. Wan, Theory and application of Hopf bifurcation, London Mathematical Society Lecture Note Series, Cambridge University Press, Cambridge (1981), doi: 10.1002/zamm.19820621221.

[4] S. Jiaqi, J. Zhujun, A new detecting method for conditions of existence of hopf bifurcation, Acta Mathematicae Applicatae Sinica, 11, No. 1 (1995), 79-93, doi: 10.1007/BF02012625. 
[5] A. Kumar, A.K. Agrawal, A. Hasan, A.K. Misra, Modeling the effect of toxicant on the deformity in a subclass of a biological species, Model. Earth Syst. Environ., 2 (2016), doi: $10.1007 / \mathrm{s} 40808-016-0086-\mathrm{x}$.

[6] Y. Kuznetsov, Elements of Applied Bifurcation Theory, New York, Springer-Verlag (2004).

[7] W. Liu, Criterion of Hopf Bifurcation without using Eigenvalues, J. Math. Anal. and App., 182 (1994), 250-256, doi: 10.1006/jmaa.1994.1079.

[8] J.B. Shukla, B. Dubey, Simultaneous effects of two toxicants on biological species: A mathematical model, J. Biol. Systems, 4 (1996), 109-130, doi: 10.1142/S0218339096000090. 